\title{
Parallel Driving in CPSS: A Unified Approach for Transport Automation and Vehicle Intelligence
}

\author{
Fei-Yue Wang, Fellow, IEEE, Nan-Ning Zheng, Fellow, IEEE, Dongpu Cao, Member, IEEE, \\ Clara Marina Martinez, Li Li, Fellow, IEEE, and Teng Liu
}

\begin{abstract}
The emerging development of connected and automated vehicles imposes a significant challenge on current vehicle control and transportation systems. This paper proposes a novel unified approach, Parallel Driving, a cloud-based cyberphysical-social systems (CPSS) framework aiming at synergizing connected automated driving. This study first introduces the CPSS and ACP-based intelligent machine systems. Then the parallel driving is proposed in the cyber-physical-social space, considering interactions among vehicles, human drivers, and information. Within the framework, parallel testing, parallel learning and parallel reinforcement learning are developed and concisely reviewed. Development on intelligent horizon (iHorizon) and its applications are also presented towards parallel horizon. The proposed parallel driving offers an ample solution for achieving a smooth, safe and efficient cooperation among connected automated vehicles with different levels of automation in future road transportation systems.
\end{abstract}

Index Terms-ACP theory, connected automated driving, cyber-physical-social systems (CPSS), iHorizon, parallel driving, parallel horizon, parallel learning, parallel reinforcement learning, parallel testing.

\section{INTRODUCTION}

A LONG with the development of cyber-physical-social systems based parallel control and management theory (ACP approach)and its applications in the past decade [1]-[7],

Manuscript received March 18, 2017; accepted May 21, 2017. Recommended by Associate Editor Mengchu Zhou. (Corresponding auth: Dongpu Cao.)

Citation: F.-Y. Wang, N.-N. Zheng, D. P. Cao, C. M. Martinez, L. Li, and T. Liu, "Parallel driving in CPSS: a unified approach for transport automation and vehicle intelligence", IEEE/CAA J. of Autom. Sinica, vol.4, no.4, pp. 577-587, Oct. 2017.

F.-Y. Wang is with the State Key Laboratory of Management and Control for Complex Systems. Institute of Automation, Chinese Academy of Sciences, China, and also with the Research Center for Military Computational Experiments and Parallel Systems Technology, National University of Defense Technology, Changsha 410073, China (e-mail: feiyue.wang@ia.ac.cn).

N.-N. Zheng is with the Institute of Artificial Intelligence and Robotics, Xi'an Jiaotong University, Xi'an 710049, China (e-mail: nnzheng@mail.xjtu.edu.cn).

D. P. Cao and C. M. Martinez are with the Advanced Vehicle Engineering Center, Cranfield University, Cranfield, MK43 0AL, UK (e-mail: d.cao@cranfield.ac.uk; c.m.marina@cranfield.ac.uk).

L. Li is with the Department of Automation, Tsinghua University, Beijing100084, China (e-mail: forcelee@gmail.com).

T. Liu is with the State Key Laboratory of Management and Control for Complex Systems, Institute of Automation, Chinese Academy of Sciences, Beijing 100190, China, and also with Qingdao Huituo Intelligent Machine Company, Qingdao, China (e-mail: tengliu17@gmail.com).

Color versions of one or more of the figures in this paper are available online at http://ieeexplore.ieee.org.

Digital Object Identifier 10.1109/JAS.2017.7510598 the framework of parallel driving has been steadily conceived, as seen in [8]-[11].This has also been significantly motivated by the emerging development in connected and automated vehicles [12]-[14].

Different levels of vehicle automation have been recently defined and recommended by SAE (2014), Germany Federal Highway Research Institute (BASt, 2012) and US National Highway Traffic Safety Administration (US NHTSA, 2013) [15]-[17], where a distinct jump in automation levels occurs between Level 2 and Level 3 automation in the SAE definition. For Level 2 automation, the driver is required to continuously monitor the driving situation, while for Level 3 the automated driving system will monitor the driving situations so the driver is allowed to be fully disengaged from the driving task. However, if requested, the driver must be ready to take over within a certain period of time.

Current automotive technology advances primarily at Level 1 and partially at Level 2, with several commercial product available, such as adaptive cruise control (ACC) for Level 1 automation, and BMW's Traffic Jam Assistant, GM's Super Cruise, Mercedes' Distronic Plus with Steering Assist, Toyota's Automated Highway Driving Assist, Volvo's ACC with Steer Assistance and Tesla Model S for Level 2 Automation [12], [18].

Thanks mainly to the DARPA Challenge, there have been substantial technological developments at Levels 4 and 5 (or full automation) [19]-[22] in the past decade, as also reflected in the Google self-driving cars. One of the on-going challenges for fully autonomous driving is the reliable and robust operation in more complex real-world driving environments, such as those found in urban driving [e.g., 13, 23]. In parallel, vehicle platoons or cooperative vehicle automation have also been investigated for a few decades, further enhancing vehicle safety, energy efficiency as well as highway capacity (e.g. the PATH project [24], along with more recent efforts [14], [25]).

European roadmap [26] has suggested three main automated driving milestones up to 2030, such that: Level 3 automation to be available at low speed and less complex driving scenarios by 2020 , and full autonomy to be available on highways by 2025 and in urban areas by 2030 that is significantly enabled by advances in connected vehicle technologies [12], [14].

It can therefore be well foreseen that in the coming two to four decades (e.g. up to 2050), our road transportation system would be consisting of a mix of connected vehicles with different levels of automation, which necessitates a unified 
approach for future smart and safe driving. This considerably motivates the development of CPSS-based parallel driving. Section II outlines CPSS and ACP-based intelligent machine framework. Section III proposes the parallel driving in CPSS. Section IV presents parallel testing, parallel learning as well as parallel reinforcement learning. Development of intelligent horizon and its applications are presented towards parallel horizon concept in Section V. Concluding remarks are then given in Section VI.

\section{CyBER-PHySiCAL-SOCIAL SPACE AND ACP-BASED INTELLIGENT MACHINE FRAMEWORK}

Cyber-physical systems (CPS) has been gaining increasing concerns in the past two decades, while CPSS augments the CPS capacity by integrating an additional dimension-human and social characteristics, so as to achieve more effective CPS design and operation [6]. This augmentation also has a philosophical implication for being in line with Karl Popper's theory of reality, which states that three interacting worlds coexist in our universe: the physical world, the mental world, and the artificial world, as shown in Fig. 1.

These three worlds are coupled by physical space and cyber space respectively, so as to conceive the cyber-physical-social space (CPSS). Rapid development in ICT enables us to exploit more in the artificial world so as to design and optimize the systems in the physical and mental world.

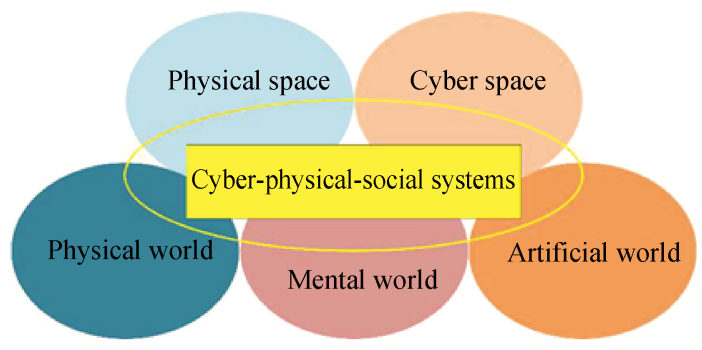

Fig. 1. Representation of cyber-physical-social spaces and their interactions.

The ACP theory and approach (Fig. 2) has been developed by Fei-Yue Wang and his research group since 2004 [1]-[7], aiming at modelling, analysis and control of complex systems, such as systems in the CPSS:

ACP $=$ Artificial societies + Computational experiments + Parallel execution

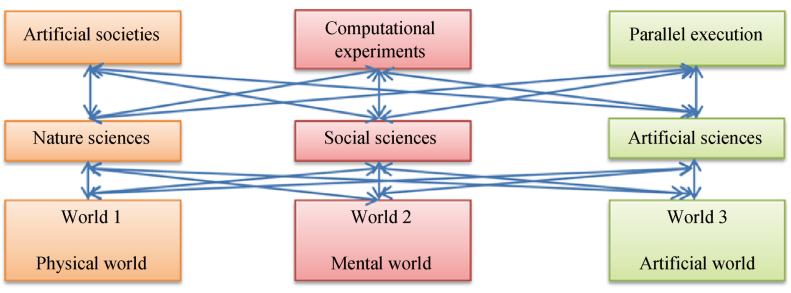

Fig. 2. Scientific foundation of the ACP approach.

Fig. 3 depicts the framework of the ACP-driven intelligent machine. In the framework, the physically-defined machine (or called Newton machine) interacts with the softwaredefined machine (or called Merton machine) through three coupled modules, namely management and control, experiment and evaluation, and learning and training, within the cyber-physical-social spaces. This parallel execution between the physically- and software-defined machines is expected to enable an optimal operation of the machines [27], [28].

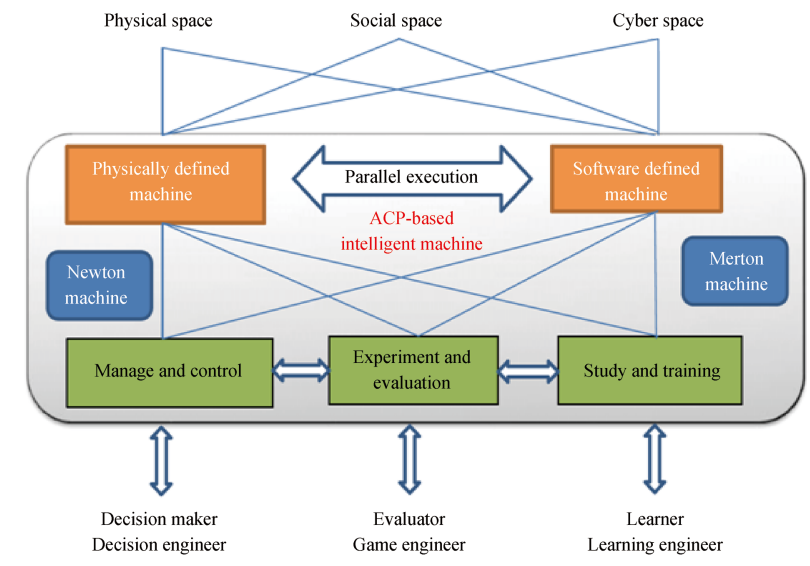

Fig. 3. Framework of ACP-based intelligent machines.

\section{PARAllel DRIVING IN CPSS}

For future connected automated driving, three main elements exists: physical vehicle (with physical attributes), human driver (with both physical (e.g. neuromuscular dynamics) and cognitive (e.g. attention, intention) attributes), as well as control and information related to driving (artificial). According to the ACP approach, these three road driving elements can be naturally projected into the three parallel worlds, namely physical world, mental world, and artificial world, as seen in Fig. 4, which conceives the CPSS-based parallel driving framework [29]-[31].

Fig. 4 presents the three levels of worlds co-existing in parallel: physical (Level I), mental (Level II), and artificial (Level III). The artificial world consists of two layers. Level IIIb refers to CPSS services consisting of three components: people (social web), place/location (geo web) and technology (sensors, Internet of Things, etc), similar to that proposed in [32]. Addition to this, in parallel driving, the artificial world is enhanced to also have a dedicated driving layer, namely Level IIIa for artificial drivers and artificial vehicles (ADAV), which is designed to realize the "computational experiments" and "parallel execution"elements within the ACP approach [7].

Furthermore, for each of the vehicles, an ADAV control module will be assigned which communicates with the artificial world and other ADAV modules, interacts with the human driver (monitoring driver state/behavior/intention, e.g., [33]-[36], joint cognition [37], driver-vehicle shared control [38]-[40], etc) in the mental world, while operating the physical vehicle in the physical world.

Human drivers are active in both mental and physical worlds, but if the fully autonomous driving function is activated, a driver's driving-related physical behaviors are not needed. However, if the driver intends to take over the control, the vehicle will switch to a lower level of automation, where driver's physical behaviors have to reengage within the driving tasks. Thus different HD-RD-ADAV units could suggest very different automation-driving patterns (e.g. from Level 0 to 

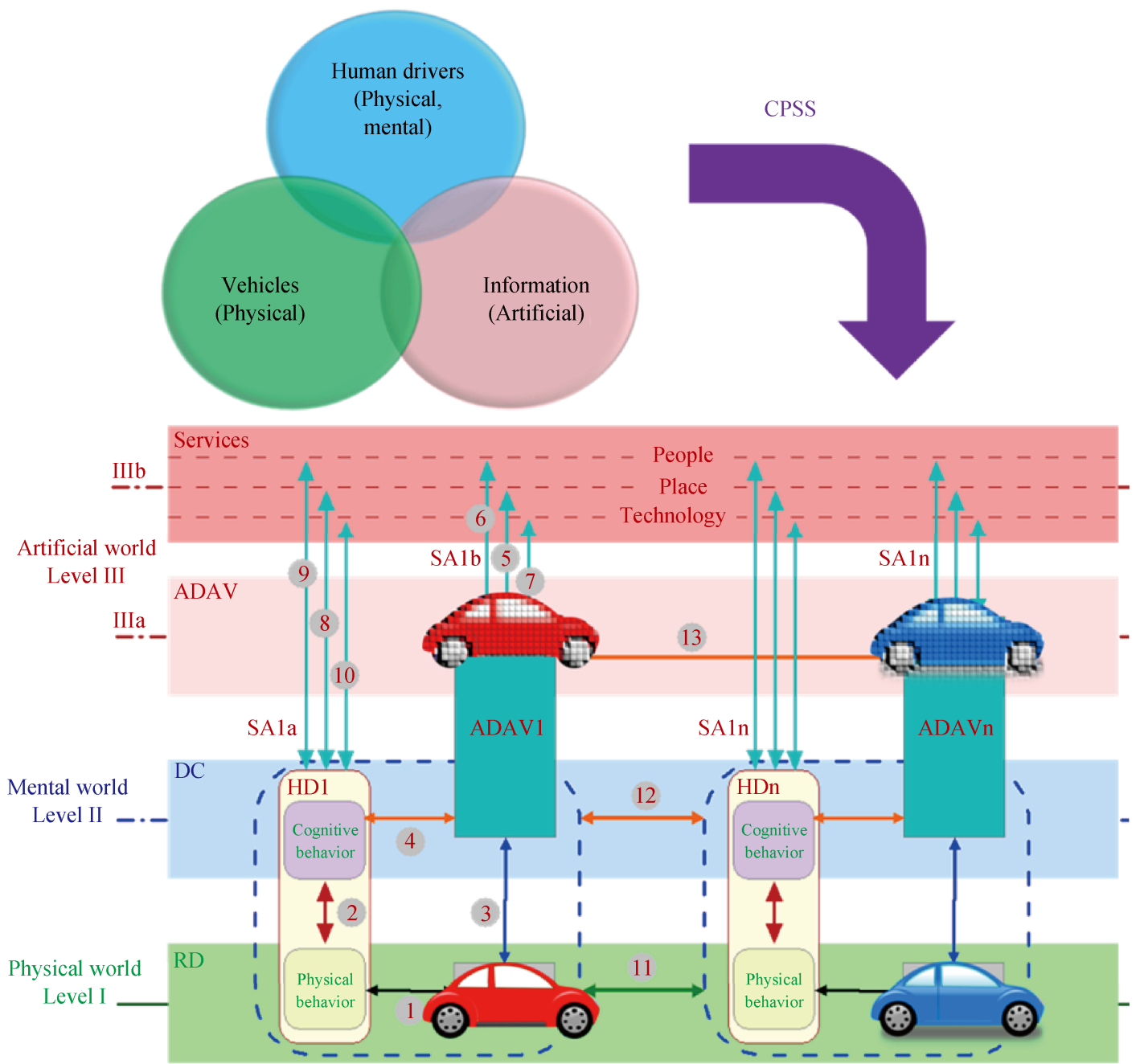

Fig. 4. Framework of parallel driving. (RD: real driving; DC: driver cognition; CPSS services including three components: people (social web), place/location (geo web), and technology (sensors, Internet of Things, etc); HD: human driver; RV: real vehicle; ADAV: artificial driver and artificial vehicle; and SA: situation awareness).

Level 6) with possible frequent shifting among different automation levels during real-world driving [41], [42].

The fundamental principle of parallel driving is that the IIIa ADAV layer together with the allocated individual ADAVs deal with the complex automated driving while keeping the real vehicles themselves as simple as possible. Fig. 5 outlines the ACP-driven synergy between physical vehicles and virtual

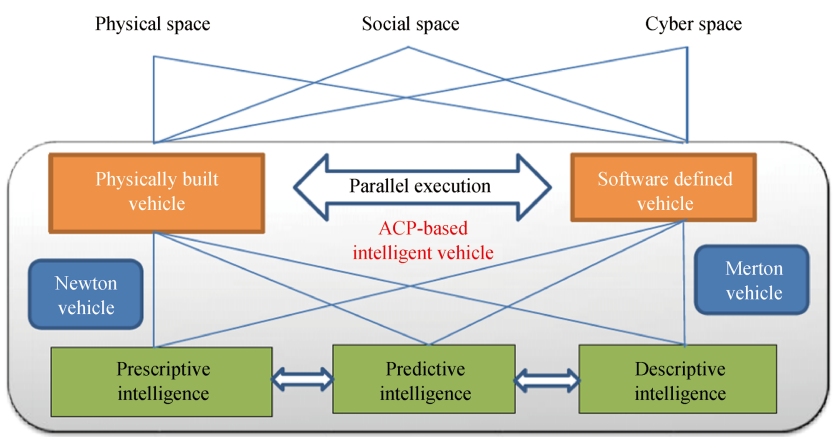

Fig. 5. ACP-based synergy between the physical built and software-defined virtual vehicles through the interactive prescriptive, predictive and descriptive intelligence within CPSS. vehicles through the interactive prescriptive, predictive and descriptive intelligence. The next session focuses on discussions on the subsystems development: parallel testing, parallel learning and parallel reinforcement learning.

\section{Parallel Testing, Parallel Learning And PARALLEL REINFORCEMENT LEARNING}

A major problem that hinders automated vehicle design is the lack of testing data. Due to the complexity of traffic scenarios, it is hardly to collect all the data to train or test the automate vehicles that we made. To address this challenge, we have proposed and developed the so called parallel learning theory in automated vehicle design [43], [44].

As shown in Fig. 6, the theoretical framework of parallel learning consists of two parts. Above the dotted line is the data preprocessing stage based on the artificial system defined by the software. The lower part of the dotted line shows the predictive learning and ensemble learning based on the computational experiment, and the parallel control and prescriptive learning. Fine arrows represent data generation or 
data learning, and bold arrows represent interactions between action and data.

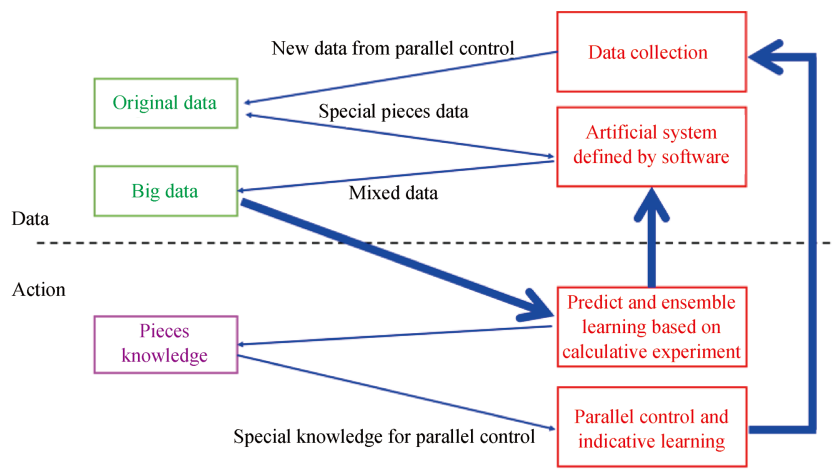

Fig. 6. Parallel learning framework.

In the data processing stage, parallel learning method first selects the specific "small data" from the original data, feeds into the software-defined artificial system, and generates a large amount of new data from the artificial system. These artificial data, together with the specific raw data, form a set of "big data" that used for updating the machine learning model. An example is that we can use GAN models to build virtual videos to test automated vehicles. Fig. 7 demonstrates our development of a simulation test platform, which includes parallel traffic systems. The upper left figure shows the cyclic updating method of the co-evolution between the real testing ground and parallel virtual testing ground, while the upper right figure presents an illustration on mapping the real view data onto virtual space for facilitating testing.

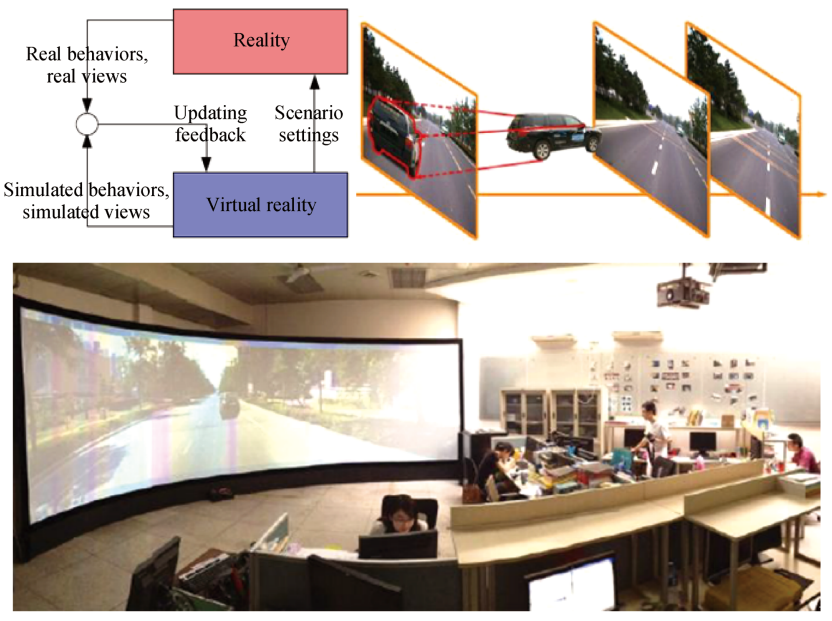

Fig. 7. Realization and demonstration of parallel testing.

Notice that we need labeled data to train automated vehicles, we use predictive learning carried out on parallel machine to self-label the data we required. Predictive learning originated from the interpretation of cognitive psychology in children's learning styles. Its core is to model the real environment inside machines, simulate and predict the possible future, and observe how the world works by observing and demonstrating. The simulation is unsupervised or semi-supervised; while the initial states and the final results are supervised.

A recently-developed example of predictive learning for parallel vehicle is "parking like human" [45]. In this example, we aims to teach automated vehicles to learn the general parking skills of human. Usually, an autonomous vehicle first plans a trajectory that links the start and destination states. Then, it determines a sequence of steering actions to make sure that the vehicle moves along with this trajectory toward the destination state. The major problem of these trajectory planning methods is that we must fully consider vehicle dynamics heavily affecting the geometry property of the candidate trajectories.

Parallel learning provides an alternative way to solve this problem by directly bridges the actual parking trajectories and the steering actions to find the best parking trajectory. First, we sample a large number of vehicle parking trajectories that we can make during a certain time period and build a deep neural network to remember all these trajectories (suppose we had shifted the start points of all these trajectories into the same original state). From the viewpoint of parallel learning, this is indeed the self-labeling process.

Specially, the input of this neural network is the destination state and the output of this neural network is the corresponding control actions and also the corresponding trajectory (Fig. 8). If more than one set of control actions can lead to the same destination state, we only let the neural network to remember the ones with optimal (time, energy, etc.) cost.Each time when a target destination state is known, we let the trained neural network to directly recall the needed control actions [46].

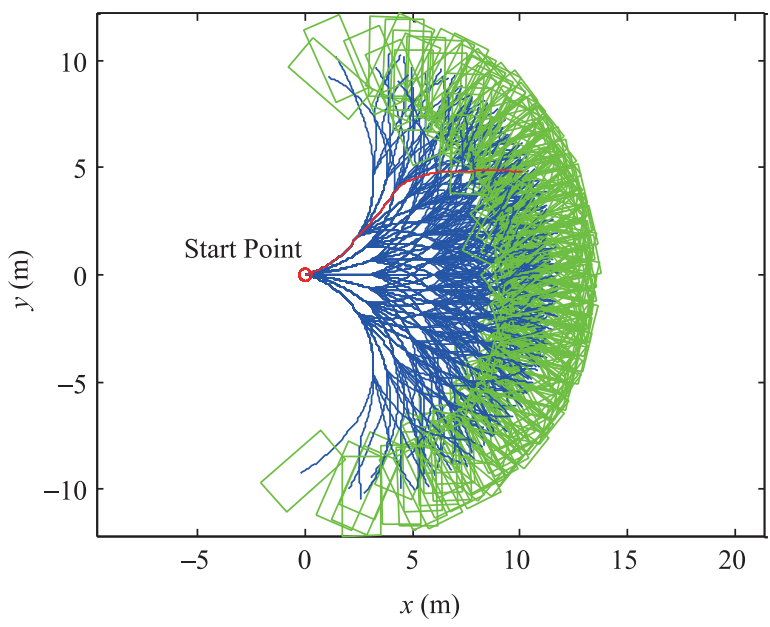

(a)

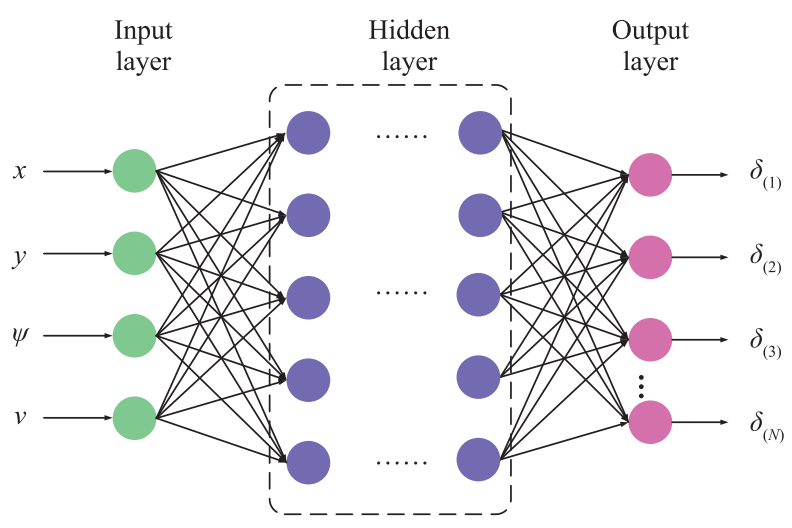

(b)

Fig. 8. Parallel parking like a human driver. 


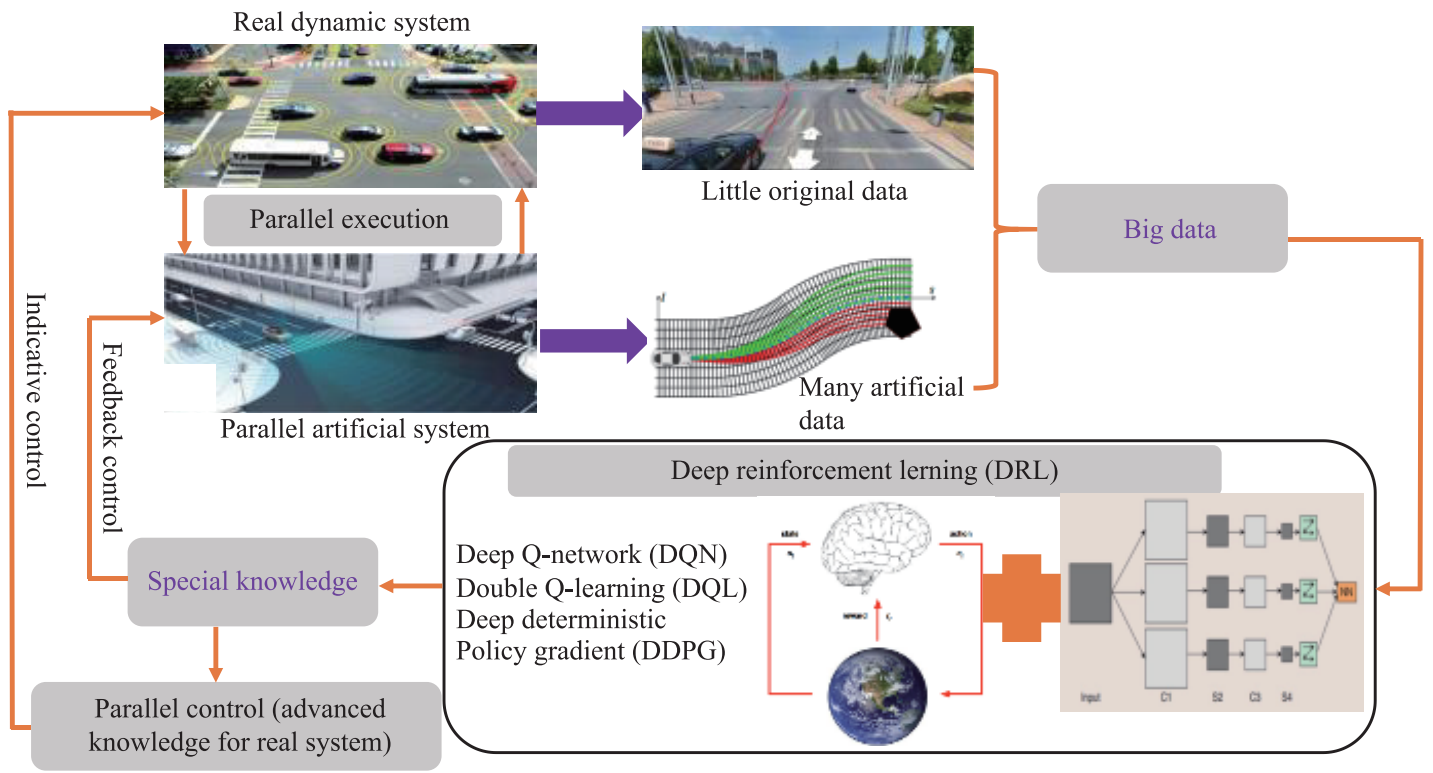

Fig. 9. The theoretical framework of the parallel reinforcement learning.

Furthermore, to handle the integrated data from the artificial system and computational experiment, we combine the parallel learning and deep reinforcement learning approaches to propose the parallel reinforcement learning (PRL) theory for automated vehicle design [47]. The framework of the PRL is depicted in Fig. 9.

In the data processing stage of deep reinforcement learning, the big data is modeled as a Markov decision process (MDP) $(\boldsymbol{S}, \boldsymbol{A}, \boldsymbol{f}, \boldsymbol{R})$, where $\boldsymbol{S}=\{s(t)\}$ and $\boldsymbol{A}=\{a(t)\}$ are set of state variables and control actions, $\boldsymbol{f}$ is the state transition probability density function, $\boldsymbol{R}=\{r(s, a)\}$ is the reward function. The optimal value function is exhibited as the finite expected discounted sum of the rewards

$$
V^{*}(s)=\min _{\pi} E\left(\sum_{t=t_{0}}^{t_{f}} \mu^{t} r(s, a)\right)
$$

where the control policy $\pi$ is the distribution over the control actions. $T=\left[t_{0}, t_{f}\right]$ is the entire time interval, $\mu \in(0,1)$ is a discount factor. To derive the optimal control action for current state variable, (1) is reformulated recursively as follow

$$
V^{*}(s)=\min _{a}\left(r(s, a)+\mu \sum_{s^{\prime} \in S} p_{s a, s^{\prime}} V^{*}\left(s^{\prime}\right)\right) \quad \forall s \in S
$$

where $p_{s a, s^{\prime}}$ is denoted as the transition probability from state $s$ to next state $s^{\prime}$ taking action $a$. As the optimal value function is determined, the optimal control policy is computed as

$$
\pi^{*}(s)=\arg \min _{a}\left(r(s, a)+\mu \sum_{s^{\prime} \in S} p_{s a, s^{\prime}} V^{*}\left(s^{\prime}\right)\right) .
$$

Furthermore, the action-value function $Q(s, a)$ and its optimal value $Q^{*}(s, a)$ are defined as the following formula:

$$
\left\{\begin{array}{l}
Q(s, a)=r(s, a)+\mu \sum_{s^{\prime} \in S} p_{s^{\prime} a, s} Q\left(s^{\prime}, a^{\prime}\right) \\
Q^{*}(s, a)=r(s, a)+\underset{s^{\prime} \in S}{\mu \sum_{s^{\prime} a, s} \min _{a^{\prime}} Q^{*}\left(s^{\prime}, a^{\prime}\right)}
\end{array}\right.
$$

In the deep Q-network method, the action-value function $Q(s, a)$ is represented by the Q-network with weights was

$$
Q_{w}(s, a)=Q^{*}(s, a) .
$$

The mean squared error (MSE) is written as

$$
I=\left(r+\mu \min _{a^{\prime}} Q_{w}\left(s^{\prime}, a^{\prime}\right)-Q_{w}(s, a)\right)^{2} .
$$

Similarly, the MSE in the double Q-learning (DQL) is rewritten as follow

$$
\begin{aligned}
I= & E_{\left(s, a, r, s^{\prime}\right)} \\
& \left\{\left(r+\mu Q_{\hat{w}}\left(s^{\prime}, \arg \min _{a^{\prime}} Q_{w}\left(s^{\prime}, a^{\prime}\right)\right)-Q_{w}(s, a)\right)^{2}\right\}
\end{aligned}
$$

in which current Q-network is used to select actions and older Q-network is used to evaluate actions.

The deep deterministic policy gradient (DDPG) is the continuous analogue of deep Q-network. The MSE in DDPG is described as

$$
I_{w}=\left(r+\mu Q_{\hat{w}}\left(s^{\prime}, \pi_{\hat{\theta}}\left(s^{\prime}\right)\right)-Q_{w}(s, a)\right)^{2} .
$$

Finally, the special knowledge derived from the PRL can be applied to the feedback control for the parallel artificial system, and also can be utilized for the indicative control in real dynamic system.

\section{Parallel Horizon: Development of Intelligent HORIZON AND IT APPLICATIONS}

Based on the current infrastructure, and with the future perspective of the simultaneous concurrence of different automation levels in common environments, the intelligent horizon (iHorizon) has been developed towards a long-term realization of parallel horizon.

iHorizon novel framework takes a step forward from current eHorizon technology made available by Bosch [48], continental [49] and HERE [50] by integrating fewer information requirements further exploited using machine learning. Furthermore, it provides a dynamic prediction of the speed profile, which can be suitable for further applications related to safety and energy consumption, and includes the driver as an essential component to improve the prognosis results. 
As shown in the Fig. 10, the baseline iHorizon consists of three main modules. First, the driving style recognition (DSR) algorithm is used to identify the driver and classify it within a continuous index into calm, normal and aggressive clusters [51], [52]. This information is used in the second and third module to provide long-term and short-term predictions of the speed profile [53], [54].

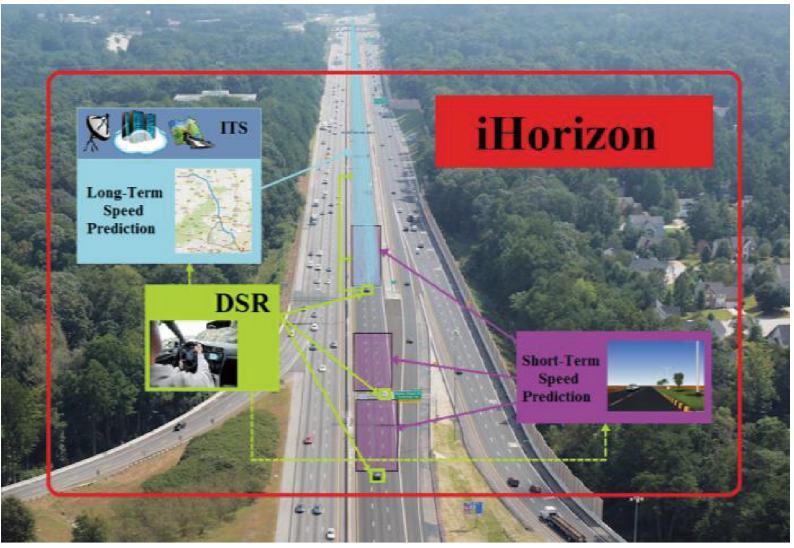

Fig. 10. The developed iHorizon framework including three main modules: 1) Driving style recognition (DSR); 2) long-term future speed prediction with cycle-length horizon; and 3) short-term future speed prediction.

\section{A. Driving Style Recognition Algorithm}

Driving style is essential to guarantee safe and efficient driving in level 0 automation vehicles, and important requirement to guarantee human acceptance of higher levels of automation [55], [56]. Eco-driving index in nowadays vehicles and driving by demonstration are some of the examples that support driving style relevance for autonomous vehicle development [57]-[60].

DSR is designed using unsupervised machine learning as introduced in [51]. Unsupervised methods allow to encourage the results generalization and support their objectivity. In the context of the iHorizon, driving style not only strongly contributes to safety improvement, but also highly affects acceleration profiles, and energy consumption [57], [58].

Fig. 11 illustrates DSR development from real-world testing data using an experimental car. The design process consist of a first stage for relevant signals selection using k-means algorithm as included in Table I and a second stage of final clusters selection and algorithm definition using Gaussian mixture model. Whilst k-means uses rigid margins to classify driving styles within calm, normal and aggressive, the Gaussians allow soft margins and provide better classification accuracy as well as consistency implementing the so-called Expectation-Maximization algorithm, as included in Table II.

Further details can be found in [49]. The data used consist of driving style specific speed profiles from rural, urban and highway roads with feedback from the driver. Unsupervised learning allows dealing with the data with independence of the subjective labels, which are only used to test the consistency of the algorithm output and the driver perception.

DSR is able to return a continuous index from 0-very calm to 1-very aggressive and provide a three class driver style classification. Driver style is used as input for future speed prediction in the second and third modules.

TABLE I

K-MEANS ALGORITHM

Step 1: Initialize $\boldsymbol{\mu}_{k}$ randomly

Step 2: Minimize $J$ with respect to $r_{n k}$ keeping $\boldsymbol{\mu}_{k}$ fixed.

The data points are classified with the current means. Calculate for all data points and means:

$r_{n k}= \begin{cases}1, & \text { if } \quad k=\arg \min _{j}\left\|x_{n}-\mu_{j}\right\|^{2} \\ 0, & \text { otherwise }\end{cases}$

Step 3: Minimize $J$ with respect to $\boldsymbol{\mu}_{k}$ keeping $r_{n k}$ fixed.

Update mean values to locate them at the minimum distance to all

elements in the cluster. Considering the total number of elements in a cluster its $n$ means are updated following:

$\frac{d J}{d \boldsymbol{\mu}_{k}}=0 \rightarrow \sum_{k=1}^{n} r_{n k}\left(\boldsymbol{x}_{n}-\boldsymbol{\mu}_{k}\right)=0 \rightarrow \boldsymbol{\mu}_{k}=\sum_{k=1}^{n} r_{n k} \boldsymbol{x}_{n} / \sum_{k=1}^{n} r_{n k}$

Repeat Steps 2 and 3 until convergence.

TABLE II

EXPECTATION-MAXIMIZATION AlgORITHM FOR GAUSSIAN MiXTURES

Step 1: Initialize parameters: $\boldsymbol{\mu}_{k}, \pi_{k}$ and $\Sigma_{k}$

Step 2: E-Expectation

Evaluate posterior probabilities with the current parameters: $\gamma\left(z_{n k}\right)$

Step 3: M-Maximization

Re-calculate the parameters using current posterior probabilities values:

$\boldsymbol{\mu}_{k}=\sum_{n=1}^{N} \gamma\left(z_{n k}\right) \boldsymbol{x}_{n} \sum_{n=1}^{N} \gamma\left(z_{n k}\right) ; \pi_{k}=N_{k} / N$

$\Sigma_{k}=\sum_{n=1}^{N} \gamma\left(z_{n k}\right)\left(\boldsymbol{x}_{n}-\boldsymbol{\mu}_{k}\right)\left(\boldsymbol{x}_{n}-\boldsymbol{\mu}_{k}\right)^{T} \sum_{n=1}^{N} \gamma\left(z_{n k}\right)$

Iterate Steps 2 and 3 until convergence of either likelihood or parameters value.

\section{B. Future Speed Prediction}

Second and third module receive diving style information and predict the future speed in cycle-length and short-term horizons. These incorporate information about the road type readily available in a commonly used GPS device, and can handle minimal information about the traffic conditions [53], [54]. In both cases, the speed and acceleration is obtained using a 2 dimensional Markov Chain which uses current speed and acceleration values to generate the next state. These consist of an array of random variables, $z$, that are conditionally independent and therefore allow for major model simplification as included in (9). The transitions are stored in a transition probability matrix (10) whose components are obtained from data with (11) [61]-[64].

$$
\begin{gathered}
P\left(z^{m+1} \mid z^{1}, \ldots, z^{m}\right)=P\left(z^{m+1} \mid z^{m}\right), \\
m \in P\{1, \ldots, M-1\} \\
T P M\left(z^{m}, z^{m+1}\right) \equiv P\left(z^{m+1} \mid z^{m}\right) \\
P_{i j}=\frac{n_{i j}}{n_{i}}, \quad n_{i}=\sum_{j=1}^{m} n_{i j}
\end{gathered}
$$




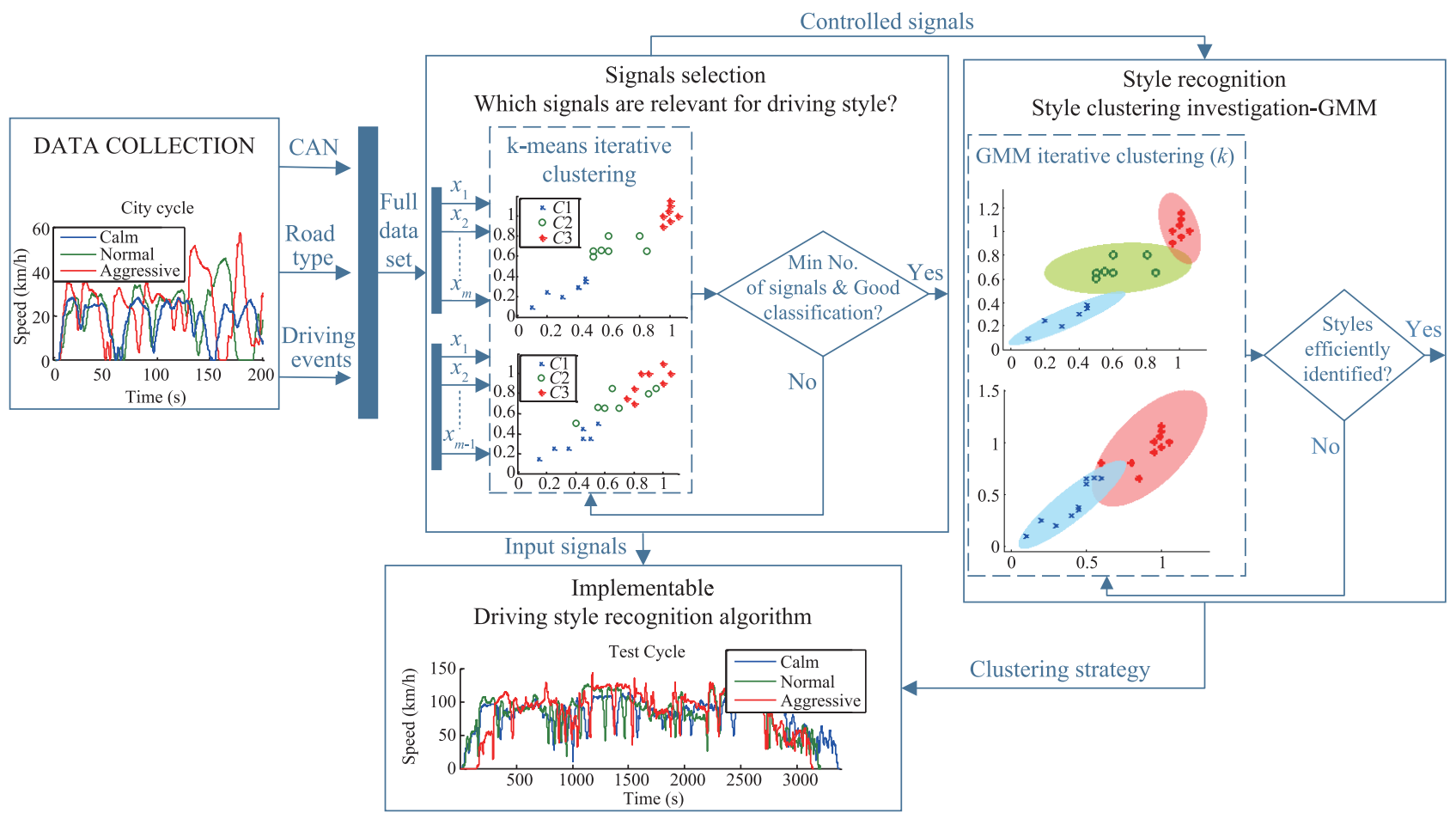

Fig. 11. Driving style recognition algorithm development using unsupervised machine learning including subjective data collection, signals selection with k-means and clusters definition with Gaussian mixture models.

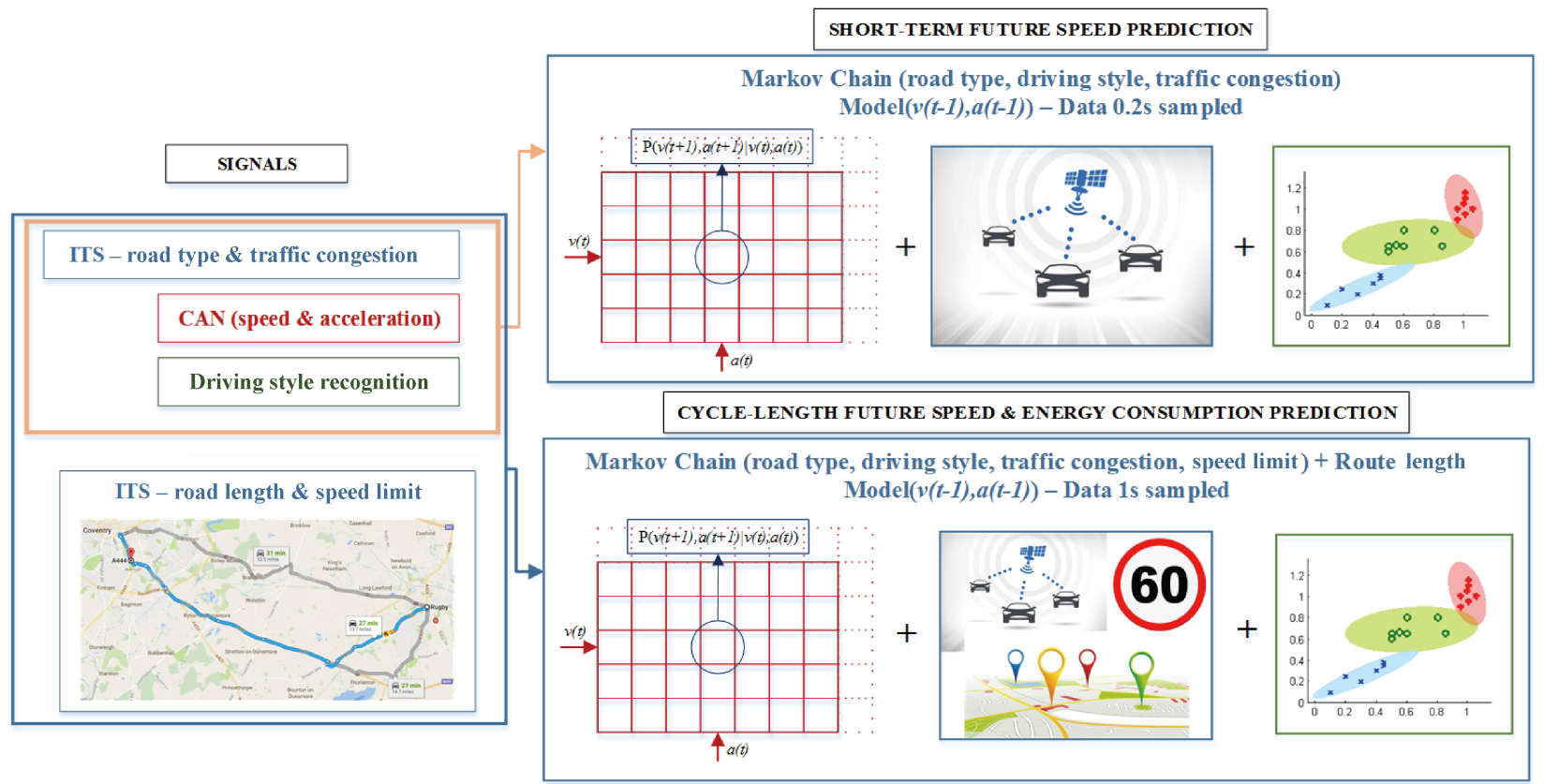

Fig. 12. Information requirement for short-term and cycle-length speed prediction. Short-term module uses a $0.2 \mathrm{~s}$ Markov Chain, driving style and road type, whilst cycle-length module incorporates the complete route, traffic information and the speed limit.

where $m$ refers to current state in time within a $M$ total states, $P$ alludes to probability function, $T P M$ is the transition probability matrix and ${ }_{n} i j$ the number of transitions observed from state $z_{i}$ to state $z_{j}$.

Cycle-length speed prediction provides a guideline speed and acceleration profile for an entire drive cycle as valuable information to anticipate energy consumption, whilst shortterm prediction is able to provide a more precise prognosis using updated speed and acceleration values, and a $5 \mathrm{~s}$ to $10 \mathrm{~s}$ window. Both models are elaborated in [53] and [54] for shortterm and cycle-length speed prediction respectively.

Fig. 12 illustrates both prediction modules along with the information requirements. The results of both cycle-length speed guideline and short-term prediction are included in Fig. 13 (left) and (right) respectively. Fig. 13 (left) illustrates short-term prediction in $10 \mathrm{~s}$ results, and long-term guideline 

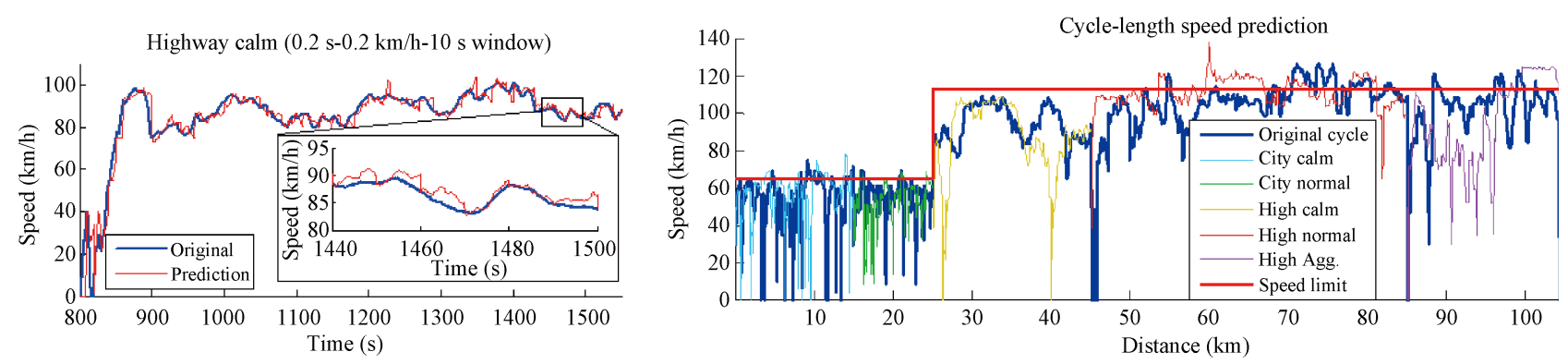

Fig. 13. (left) shot-term speed prediction in highway road with a calm driver; (right) cycle-length guideline speed profile for a complete cycle combining city and highway driving with variable driving style.

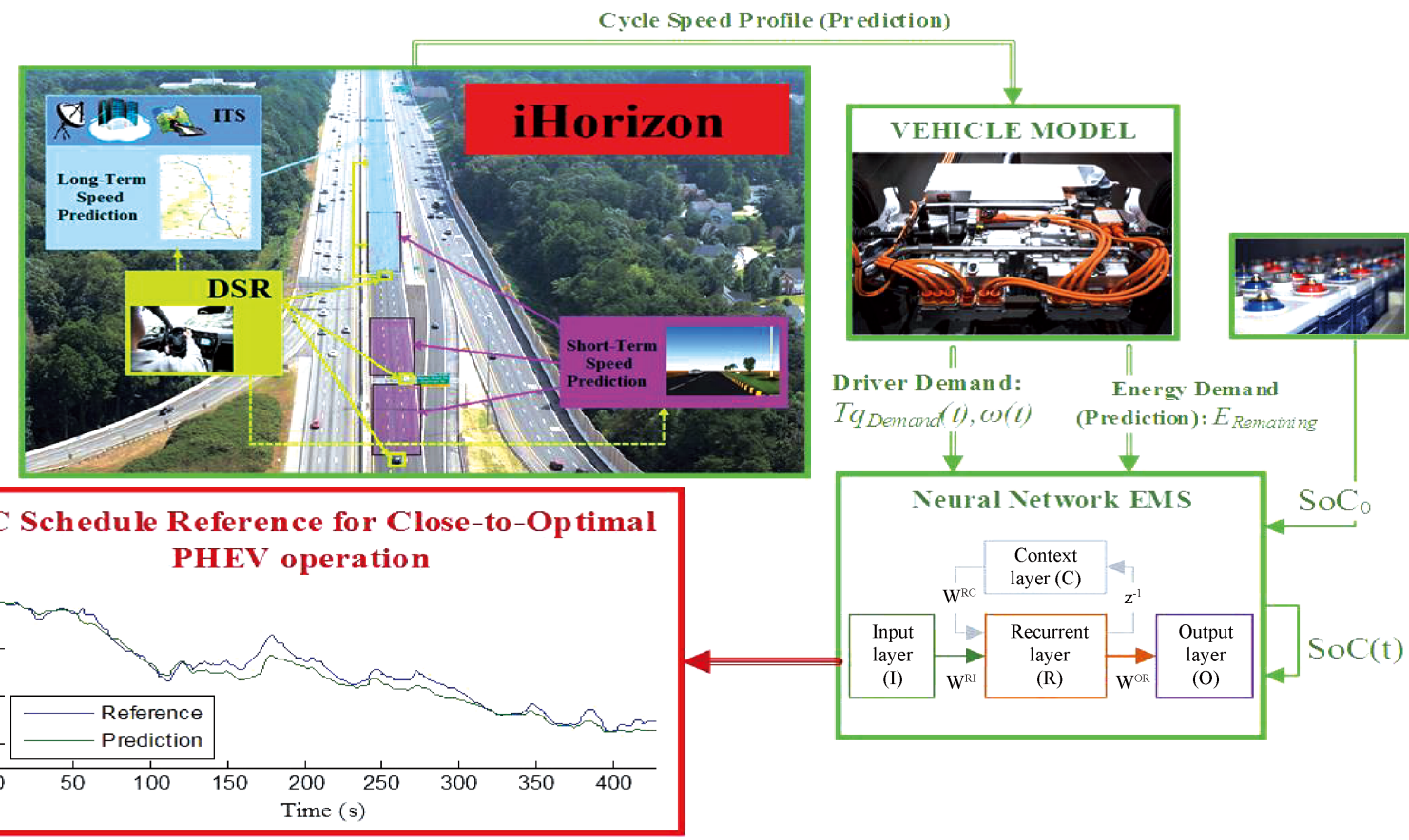

Fig. 14. Intelligent energy management for PHEV based on iHorizon application and neural network control.

profile is included in Fig. 13 (right).

\section{C. iHorizon Application to Intelligent Energy Management}

iHorizon framework allows for multiple application in terms for energy management and autonomous feature development. Probably one of the most evident is the use of the cycle-length speed prediction for plug-in hybrid electric vehicle (PHEV) control optimization. Both acceleration and speed profile can be used along with a vehicle model to anticipate driver power demand and cycle power requirements so as to guarantee full battery depletion by the end of the planned cycle. This is the case explained in [61] where the authors use multiple optimal control strategies, which produced using dynamic programming, to teach a layer recurrent neural network using back propagation, where the weights update is modified using extended Kalman filter (EKF). In this approach to neural network training the weights update is described as a finding the minimum square error of the weights estimation as in (12) using the EKF algorithm included in (13)-(15).

$$
\left\{\begin{array}{l}
\omega(t+1)=\omega(t)+q(t) \\
d(t)=h(\omega(t))+r(t)
\end{array}\right.
$$

$$
K(t)=P(t) H(t)\left[R(t)+H(t)^{T} P(t) H(t)\right]^{-1}
$$

$$
\begin{gathered}
\hat{\omega}(t+1)=\hat{\omega}(t)(d(t)-H(t) \hat{\omega}(t)) \\
P(t+1)=P(t)-K(t) H(t)^{T} P(t)+Q(t)
\end{gathered}
$$

where $\omega, \hat{\omega}_{k}, P_{k}, R_{k}, H_{k}$, and $Q_{k}$ are respectively: weights value, weights estimation using EKF, error covariance matrix, measurement noise covariance matrix, matrix derivative with respect to the weights of the network outputs and process noise covariance matrix [65]-[67].

The intelligent energy management resulting from the neural network training makes uses of the iHorizon as illustrated in Fig. 14 and is able to emulate with an average 3\% state of charge error optimal results with potential real time capability. The results in term of fuel consumption where also very optimistic in simulation environment event for reasonable long drive cycles with a maximum absolute error below $0.06 \mathrm{~kg}$ in all cases (Fig. 15). 

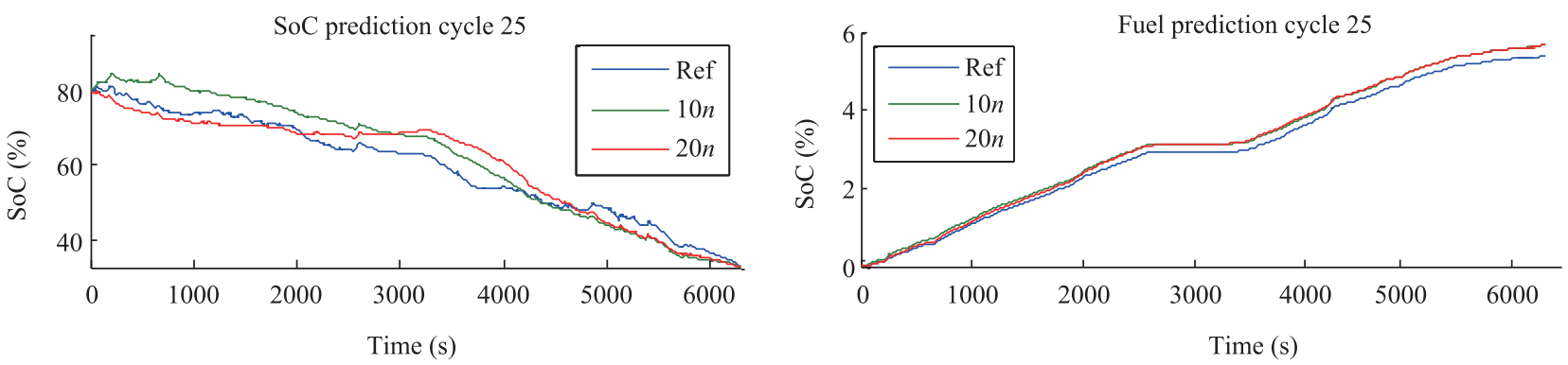

Fig. 15. (left) SoC error with respect to the global optimal reference (blue) using 10 and 20 neurons in the hidden layer; (right) idem for fuel consumption error.

\section{CONCLUDING REMARKS}

Based on the ACP approach, this paper proposes a novel cloud-based CPSS parallel driving framework for synergizing future connected automated driving involving complex multilevel vehicle automation as well as driver-automation interactions. Within the parallel driving framework, parallel testing, parallel learning, parallel reinforcement learning, as well as development of intelligent horizon (iHorizon) towards parallel horizon are presented and discussed with examples.

The fundamental principle of parallel driving is that the designed computational artificial ADAV layer together with the allocated individual ADAV control modules deal with the complex automated driving while keeping the real vehicles as simple as possible. The proposed parallel driving is able to offer an ample solution for achieving a smooth, safe and efficient collaboration among connected automated vehicles with different levels of automation in future transportation.

\section{REFERENCES}

[1] F. Y. Wang, "Artificial societies, computational experiments, and parallel systems: A discussion on computational theory of complex socialeconomic systems," Complex Syst. Complexity Sci., vol. 1, no. 4, pp. 25-35, Oct. 2004.

[2] F. Y. Wang, "Parallel system methods for management and control of complex systems," Control Decision, vol. 19, no. 5, pp. 485-489, 514, May 2004

[3] F. Y. Wang, "Agent-based control for networked traffic management systems," IEEE Intell. Syst., vol. 20, no. 5, pp. 92-96, Sept.-Oct. 2005.

[4] F. Y. Wang, "Toward a revolution in transportation operations: AI for complex systems," IEEE Intell. Syst., vol. 23, no. 6, pp. 8-13, Nov.-Dec. 2008

[5] N. Zhang, F. Y. Wang, F. H. Zhu, D. B. Zhao, and S. M. Tang, "DynaCAS: Computational experiments and decision support for ITS," IEEE Intell. Syst., vol. 23, no. 6, pp. 19-23, Nov.-Dec. 2008.

[6] F. Y. Wang, "The emergence of intelligent enterprises: From CPS to CPSS," IEEE Intell. Syst., vol. 25, no. 4, pp. 85-88, Jul.-Aug. 2010.

[7] F. Y. Wang, "Parallel control and management for intelligent transportation systems: Concepts, architectures, and applications," IEEE Trans. Intell. Transp. Syst., vol. 11, no. 3, pp. 630-638, Sep. 2010.

[8] F. Y. Wang, "Agent-based control strategies for smart and safe vehicles," in Proc. IEEE International Conference on Vehicular Electronics and Safety, Shaan'xi, China, 2005, pp. 331-332.

[9] F. Y. Wang, "Scanning the issue and beyond: Parallel driving with software vehicular robots for safety and smartness," IEEE Trans. Intell. Transp. Syst., vol. 15, no. 4, pp. 1381-1387, Aug. 2014.

[10] L. Li, Y. L. Lin, D. P. Cao, N. N. Zheng, and F. Y. Wang, "Parallel learning-a new framework for machine learning," Acta Automat. Sin. vol. 43, no. 1, pp. 1-18, Jan. 2017.
[11] T. X. Bai, S. Wang, Z. Shen, D. P. Cao, N. N. Zheng, and F. Y. Wang, "Parallel robotics and parallel unmanned systems: Framework, structure, process, platform and applications," Acta Automat. Sin., vol. 43, no. 2, pp. $161-175$, Feb. 2017.

[12] K. Bengler, K. Dietmayer, B. Farber, M. Maurer, C. Stiller, and H. Winner, "Three decades of driver assistance systems: Review and future perspectives," IEEE Intell. Transp. Syst. Mag., vol. 6, no. 4, pp. 6-22, 2014.

[13] X. H. Li, Z. P. Sun, D. P. Cao, Z. He, and Q. Zhu, "Real-time trajectory planning for autonomous urban driving: Framework, algorithms, and verifications," IEEE/ASME Trans. Mech., vol. 21, no. 2, pp. 740-753, Apr. 2016.

[14] Y. Zheng, S. B. Li, J. Q. Wang, D. P. Cao, and K. Q. Li, "Stability and scalability of homogeneous vehicular platoon: Study on the influence of information flow topologies," IEEE Trans. Intell. Transp. Syst., vol. 17, no. 1, pp. 14-26, Jan. 2016.

[15] Taxonomy and Definitions for Terms Related to On-road Motor Vehicle Automated Driving Systems, SAE J3016, 2014.

[16] T. M. Gasser and D. Westhoff, "BASt-study: Definitions of automation and legal issues in Germany," in Proc. 2012 Road Vehicle Automation Workshop, 2012.

[17] National Highway Traffic Safety Administration (NHTSA), "Preliminary Statement of Policy Concerning Automated Vehicles," USA, 2013.

[18] J. C. de Winter, R. Happee, M. H. Martens, and N. A. Stanton, "Effects of adaptive cruise control and highly automated driving on workload and situation awareness: A review of the empirical evidence," Transp. Res. F: Traffic Psychol. Behav., vol. 27, pp. 196-217, Nov. 2014.

[19] T. Luettel, M. Himmelsbach, and H. J. Wuensche, "Autonomous ground vehicles-concepts and a path to the future," Proc. IEEE, vol. 100, pp. 1831-1839, May 2012

[20] M. Aeberhard, S. Rauch, M. Bahram, G. Tanzmeister, J. Thomas, Y. Pilat, F. Homm, W. Huber, and N. Kaempchen, "Experience, results and lessons learned from automated driving on Germany's highways," IEEE Intell. Transp. Syst. Mag., vol. 7, no. 1, pp. 42-57, 2015.

[21] C. Urmson, J. Anhalt, D. Bagnell, C. Baker, R. Bittner, M. N. Clark, J. Dolan, D. Duggins, T. Galatali, C. Geyer, M. Gittleman, S. Harbaugh, M. Hebert, T. M. Howard, S. Kolski, A. Kelly, M. Likhachev, M. McNaughton, N. Miller, K. Peterson, B. Pilnick, R. Rajkumar, P. Rybski, B. Salesky, Y. W. Seo, S. Singh, J. Snider, A. Stentz, W. Red Whittaker, Z. Wolkowicki, J. Ziglar, H. Bae, T. Brown, D. Demitrish, B. Litkouhi, J. Nickolaou, V. Sadekar, W. Zhang, J. Struble, M. Taylor, M. Darms, and D. Ferguson, "Autonomous driving in urban environments: Boss and the urban challenge," J. Field Rob., vol. 25, no. 8, pp. 425-466, Aug. 2008 .

[22] J. R. McBride, J. C. Ivan, D. S. Rhode, J. D. Rupp, M. Y. Rupp, J. D. Higgins, D. D. Turner, and R. M. Eustice, "A perspective on emerging automotive safety applications, derived from lessons learned through participation in the darpa grand challenges," J. Field Rob., vol. 25, no. 10, pp. 808-840, Oct. 2008.

[23] F. Havlak and M. Campbell, "Discrete and continuous, probabilistic anticipation for autonomous robots in urban environments," IEEE Trans. Rob., vol. 30, no. 2, pp. 461-474, Apr. 2014. 
[24] P. Seiler, A. Pant, and J. K. Hedrick, "Disturbance propagation in vehicle strings," IEEE Trans. Autom. Control, vol. 49, no. 10, pp. 1835-1842, Oct. 2004.

[25] S. E. Li, F. Gao, D. P. Cao, and K. Q. Li, "Multiple-model switching control of vehicle longitudinal dynamics for platoon-level automation," IEEE Trans. Veh. Technol., vol. 65, no. 6, pp. 4480-4492, Jun. 2016.

[26] J. Dokic, B. Müller, and G. Meyer, "European roadmap: Smart systems for automated driving," 2015.

[27] K. F. Wang, C. Gou, and F. Y. Wang, "Parallel vision: an ACP-based approach to intelligent vision computing," Acta Automat. Sin., vol. 42, no. 10 , pp. $1490-1500$, Oct. 2016.

[28] K. F. Wang, C. Gou, N. N. Zheng, J. M. Rehg, and F. Y. Wang, "Parallel vision for perception and understanding of complex scenes: methods, framework, and perspectives," Artif. Intell. Rev., vol. 48, no. 3, pp. $298-328$, Oct. 2017.

[29] Y. Liu, Y. Peng, B. L. Wang, S. R. Yao, and Z. H. Liu, "Review on cyber-physical systems," IEEE/CAA J. Automat. Sin., vol. 4, no. 1, pp. 27-40, Jan. 2017.

[30] X. P. Guan, B. Yang, C. L. Chen, W. B. Dai, and Y. Y. Wang, "A comprehensive overview of cyber-physical systems: From perspective of feedback system," IEEE/CAA J. Automat. Sin., vol. 3, no. 1, pp. 1-14, Jan. 2016.

[31] F. Y. Wang, "Control 5.0: From newton to merton in popper's cybersocial-physical spaces," IEEE/CAA J. Automat. Sin., vol. 3, no. 3, pp. 233-234, Jul. 2016.

[32] R. Schroeter, A. Rakotonirainy, and M. Foth, "The social car: new interactive vehicular applications derived from social media and urban informatics," in Proc. 4th International Conference on Automotive User Interfaces and Interactive Vehicular Applications, Portsmouth, New Hampshire, 2012, pp. 107-110.

[33] J. F. Coughlin, B. Reimer, and B. Mehler, "Monitoring, managing, and motivating driver safety and well-being," IEEE Perv. Comput., vol. 10, no. 3, pp. 14-21, Jul.-Sep. 2011

[34] L. Li, D. Wen, N. N. Zheng, and L. C. Shen, "Cognitive cars: A new frontier for ADAS research," IEEE Trans. Intell. Transp. Syst., vol. 13, no. 1, pp. 395-407, Mar. 2012.

[35] C. Ahlstrom, K. Kircher, C. Fors, T. Dukic, C. Patten, and A. Anund, "Measuring driver impairments: Sleepiness, distraction, and workload," IEEE Pulse, vol. 3, no. 2, pp. 22-30, Mar. 2012.

[36] A. Pentland and A. Liu, "Modeling and prediction of human behavior," Neural Comput., vol. 11, no. 1, pp. 229-242, Jan. 1999.

[37] D. B. Miller and W. Ju, "Joint cognition in automated driving: combining human and machine intelligence to address novel problems," in 2015 AAAI Spring Symposium Series, 2015.

[38] L. Saleh, P. Chevrel, F. Claveau, J. F. Lafay, and F. Mars, "Shared steering control between a driver and an automation: Stability in the presence of driver behavior uncertainty," IEEE Trans. Intell. Transp. Syst., vol. 14, no. 2, pp. 974-983, Jun. 2013.

[39] X. X. Na and D. J. Cole, "Linear quadratic game and non-cooperative predictive methods for potential application to modelling driver-AFS interactive steering control," Veh. Syst. Dyn., vol. 51, no. 2, pp. 165-198, Feb. 2013.

[40] F. O. Flemisch, K. Bengler, H. Bubb, H. Winner, and R. Bruder, "Towards cooperative guidance and control of highly automated vehicles: H-mode and conduct-by-wire," Ergonomics, vol. 57, no. 3, pp. 343-360, Feb. 2014

[41] F. Y. Wang, J. Zhang, Q. L. Wei, X. H. Zheng, and L. Li, "PDP: Parallel dynamic programming," IEEE/CAA J. Automat. Sin., vol. 4, no. 1, pp. $1-5$, Jan. 2017

[42] F. Y. Wang, X. Wang, L. X. Li, and L. Li, "Steps toward parallel intelligence," IEEE/CAA J. Automat. Sin., vol. 3, no. 4, pp. 345-348, Oct. 2016.

[43] L. Li, Y. L. Lin, D. P. Cao, N. N. Zheng, and F. Y. Wang, "Parallel learning-a new framework for machine learning," Acta Automat. Sin., vol. 43, pp. 1-18, no. 1, Jan. 2017.
[44] L. Li, Y. L. Lin, N. N. Zheng, and F. Y. Wang, "Parallel learning: a perspective and a framework," IEEE/CAA J. Automat. Sin., vol. 4, no. 3, pp. 389-395, 2017.

[45] W. Liu, Z. Li, L. Li, and F. Y. Wang, "Parking like a human: A direct trajectory planning solution," IEEE Trans. Intell. Transp. Syst., 2017, doi: 10.1109/TITS.2017.2687047.

[46] M. Z. Kang and F. Y. Wang, "From parallel plants to smart plants: Intelligent control and management for plant growth," IEEE/CAA J. Autom. Sin., vol. 4, no. 2, pp. 161-166, Apr. 2017.

[47] F. Y. Wang, J. J. Zhang, X. H. Zheng, X. Wang, Y. Yuan, X. X. Dai, J. Zhang, and L. Q. Yang, "Where does AlphaGo go: From church-turing thesis to AlphaGo thesis and beyond," IEEE/CAA Journal of Automatica Sinica, vol. 3, no. 2, pp. 113-120, Apr. 2016.

[48] http://www.bosch-mobility-solutions.com/en/products-andservices/commercial-vehicles/connectivity-solutions/connectivity/ electronic-horizon/. Accessed: Feb. 2, 2017.

[49] http://www.continental-automotive.com/www/automotive_de_en/themes/ passenger_cars/interior/connectivity/pi_ehorizon_en.html [Online]. Accessed: Feb. 2, 2017

[50] https://here.com/en/products-services/products/here-electronic-horizon [Online]. Accessed: Feb. 2, 2017.

[51] C. Marina Martinez, D. P. Cao, E. Velenis, D. Clarke, B. Gao, and M. Wellers, "Driving style recognition for driver-automation collaboration using unsupervised learning," IEEE Trans. Hum.-Mach. Syst., under review.

[52] C. Marina Martinez, M. Heucke, F. Y. Wang, B. Gao, D. Cao, and E. Velenis, "Driving style recognition for intelligent vehicle control and advanced driver assistance: A survey," IEEE Trans. Intell. Transp. Syst., in press, 2017.

[53] C. Marina Martinez, B. I. Kim, D. P. Cao, E. Velenis, and B. Gao, "Intelligent Horizon Part I: Driving-Style-Enabled Short-Term Future Speed Prediction," IEEE Trans. Intell. Transp. Syst., under review.

[54] C. Marina Martinez, B. I. Kim, D. Cao, E. Velenis, and B. Gao, "Intelligent Horizon Part II: Driving-Style-Enabled Cycle-Term Future Speed Prediction," IEEE Trans. Intell. Transp. Syst., under review.

[55] A. L. Kun, S. Boll, and A. Schmidt, "Shifting gears: User interfaces in the age of autonomous driving," IEEE Perv. Comput., vol. 15, No. 1 , pp. 32-38, Jan.-Mar. 2016

[56] K. Bengler, K. Dietmayer, B. Farber, M. Maurer, C. Stiller, and H. Winner, "Three decades of driver assistance systems: Review and future perspectives," IEEE Intell. Transp. Syst. Mag., vol. 6, no. 4, pp. 6-22, 2014.

[57] F. Syed, S. Nallapa, A. Dobryden, C. Grand, R. McGee, and D. Filev, "Design and analysis of an adaptive real-time advisory system for improving real world fuel economy in a hybrid electric vehicle," SAE, SAE Technical Paper 2010-01-0835, 2010.

[58] V. Manzoni, A. Corti, P. De Luca, and S. M. Savaresi, "Driving style estimation via inertial measurements," in Proc. 2010 13th International IEEE Conference in Intelligent Transportation Systems, Funchal, Portugal, 2010, pp. 777-782.

[59] T. Y. Gu and J. M. Dolan, "Toward human-like motion planning in urban environments," in Proc. 2014 IEEE Intelligent Vehicles Symposium, Dearborn, MI, USA, 2014, pp. 350-355.

[60] M. Kuderer, S. Gulati, and W. Burgard, "Learning driving styles for autonomous vehicles from demonstration," in Proc. 2015 IEEE International Conference on Robotics and Automation, Seattle, WA, USA, 2015, pp. 2641-2646.

[61] C. Marina Martinez, D. P. Cao, B. Velenis, and B. Gao, "Intelligent Energy Management for Plug-in Hybrid Electric Vehicles Using Neural Networks and The Intelligent Horizon," submitted to IEEE Transactions on Control Systems Technology.

[62] C. M. Bishop, Pattern Recognition and Machine Learning. New York, USA: Springer, 2006

[63] K. P. Murphy, Machine Learning: A Probabilistic Perspective. Cambridge, USA: The MIT Press, 2012. 
[64] T. W. Anderson and L. A. Goodman, "Statistical inference about Markov chains," Ann. Math. Stat., vol. 28, no. 1, pp. 89-110, 1957.

[65] G. V. Puskorius and L. A. Feldkamp, "Parameter-based Kalman filter training: theory and implementation," in Kalman Filtering and Neural Networks, S. Haykin, Ed. New York, USA: John Wiley \& Sons, Inc., 2001.

[66] Q. L. Wei, D. R. Liu, Y. Liu, and R. Z. Song, "Optimal constrained self-learning battery sequential management in microgrid via adaptive dynamic programming," IEEE/CAA J. Automat. Sin., vol. 4, no. 2, pp. 168-176, Apr. 2017.

[67] D. Yu and J. Y. Li, "Recent progresses in deep learning based acoustic models," IEEE/CAA J. Automat. Sin., vol. 4, no. 3, pp. 396-409, 2017.

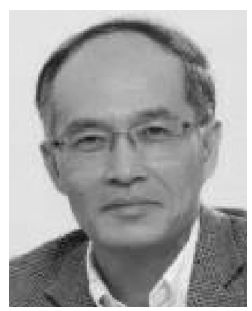

Fei-Yue Wang (S'87-M'89-SM'94-F'03) received his $\mathrm{Ph} . \mathrm{D}$. in Computer and Systems Engineering from Rensselaer Polytechnic Institute, Troy, New York in 1990. He joined the University of Arizona in 1990 and became a Professor and Director of the Robotics and Automation Lab (RAL) and Program in Advanced Research for Complex Systems (PARCS). In 1999, he founded the Intelligent Control and Systems Engineering Center at the Institute of Automation, Chinese Academy of Sciences (CAS), Beijing, China, under the support of the Outstanding Overseas Chinese Talents Program from the State Planning Council and "100 Talent Program" from CAS, and in 2002, was appointed as the Director of the Key Lab of Complex Systems and Intelligence Science, CAS. From 2006 to 2010, he was Vice President for Research, Education, and Academic Exchanges at the Institute of Automation, CAS. In 2011, he became the State Specially Appointed Expert and the Director of the State Key Laboratory of Management and Control for Complex Systems.

Dr. Wang's research focuses on methods and applications for parallel systems, social computing, and knowledge automation. He was the Founding Editor-in-Chief of the International Journal of Intelligent Control and Systems (1995-2000), Founding EiC of IEEE ITS Magazine (2006-2007), EiC of IEEE Intelligent Systems (2009-2012), and EiC of IEEE Transactions on ITS (2009-2016). Currently he is EiC of IEEE Transactions on Computational Social Systems, Founding EiC of IEEE/CAA Journal of Automatica Sinica, and Chinese Journal of Command and Control. Since 1997, he has served as General or Program Chair of more than 20 IEEE, INFORMS, ACM, and ASME conferences. He was the President of IEEE ITS Society (20052007), Chinese Association for Science and Technology (CAST, USA) in 2005, the American Zhu Kezhen Education Foundation (2007-2008), and the Vice President of the ACM China Council (2010-2011). Since 2008, he has been the Vice President and Secretary General of Chinese Association of Automation. Dr. Wang has been elected as Fellow of IEEE, INCOSE, IFAC, ASME, and AAAS. In 2007, he received the National Prize in Natural Sciences of China and was awarded the Outstanding Scientist by ACM for his research contributions in intelligent control and social computing. He received IEEE ITS Outstanding Application and Research Awards in 2009, 2011 and 2015, and IEEE SMC Norbert Wiener Award in 2014.

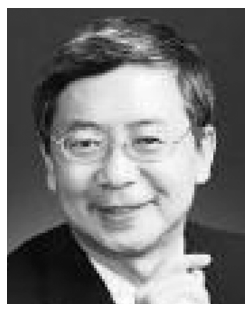

Nan-Ning Zheng (SM'93-F'06) graduated from the Department of Electrical Engineering, Xi'an Jiaotong University, Xi'an, China, in 1975, and received the M.S. degree in information and control engineering from Xi'an Jiaotong University in 1981, and the Ph.D. degree in electrical engineering from Keio University, Yokohama, Japan, in 1985. He joined Xi'an Jiaotong University in 1975, and he is currently a Professor and the Director of the Institute of Artificial Intelligence and Robotics. His research interests include computer vision, pattern recognition and image processing, and hardware implementation of intelligent systems. Prof. Zheng became a member of the Chinese Academy of Engineering in 1999, and he is the Chinese Representative on the Governing Board of the International Association for Pattern Recognition. He also serves as the President of Chinese Association of Automation.

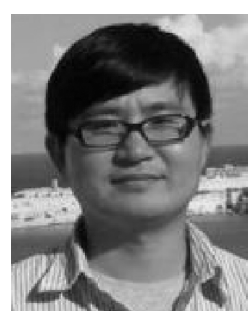

Dongpu Cao received the Ph.D. degree from Concordia University, Canada, in 2008. He is currently a Senior Lecturer at the Advanced Vehicle Engineering Center, Cranfield University, UK. His research focuses on vehicle dynamics/control and intelligence, automated driving and parallel driving, where he has contributed more than 120 publications and 1 US patent. He received the ASME AVTT'2010 Best Paper Award and 2012 SAE Arch T. Colwell Merit Award. Dr. Cao serves as an Associate Editor for IEEE Transactions on Intelligent Transportation Systems, IEEE Transactions on Vehicular Technology and IEEE Transactions on Industrial Electronics. He has been a Guest Editor for Vehicle System Dynamics, IEEE/ASME Transactions on Mechatronics and IEEE Transactions on Human-Machine Systems. He has been serving on the SAE International Vehicle Dynamics Standards Committee and a few ASME, SAE, IEEE technical committees.

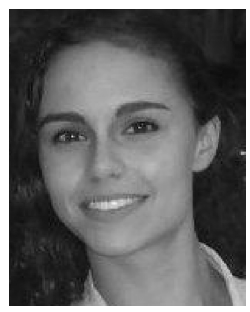

Clara Marina Martinez received her Ph.D. degree at Advance Vehicle Engineering Centre, Cranfield University, UK, in 2017. She obtained the 5-years degree in Industrial Engineering from Seville University, Spain, and the MSc degree in automotive mechatronics from Cranfield University in 2014. She will be joining Porsche Engineering R\&D Center, Germany, in Nov. 2017. Her current research focuses on intelligent control for electrified vehicles, driving style recognition, future speed prediction, and machine learning. She has published over 10 papers.

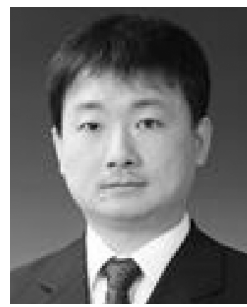

Li Li (S'05-M'06-SM'10-F'16) is currently an Associate Professor with the Department of Automation, Tsinghua University, Beijing, China, working in the fields of complex and networked systems, intelligent control and sensing, intelligent transportation systems, and intelligent vehicles. Dr. Li had published more than 50 SCI indexed international journal papers and more than 50 international conference papers as a first/corresponding author. He serves as an Associate Editor of IEEE Transactions on Intelligent Transportation Systems.

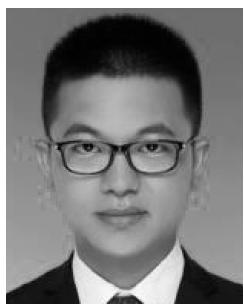

Teng Liu received the B.S. degree in mathematics from Beijing Institute of Technology, Beijing, China, 2011. He received his Ph.D. degree in the vehicle engineering from Beijing Institute of Technology (BIT), Beijing, in 2017. Dr. Liu is now a postdoctoral research fellow at the Institute of Automation, Chinese Academy of Sciences, China. His current research focuses on parallel driving, parallel reinforcement learning, automated driving, and energy management of electrified vehicles. $\mathrm{He}$ has published over 20 papers in these areas. 
2017-09-15

Parallel driving in CPSS: a unified approach for transport automation and vehicle intelligence

Wang, Fei-Yue

IEEE

Wang F, Zheng N, Cao D, Martinez C. Li L, Liu T, Parallel driving in CPSS: A unified approach for transport automation and vehicle intelligence, IEEE Caa Journal of Automatica Sinica, Vol. 4, Issue 4, 2017, pp. 577-587

http://dx.doi.org/10.1109/JAS.2017.7510598

Downloaded from Cranfield Library Services E-Repository 